\title{
Análisis de la docencia universitaria por la influencia de la COVID-19: algunas lecciones aprendidas Analysis of the university teaching due to the influence of COVID-19: some lessons learned
}

\author{
Amez I., Castells B., Sánchez-Canales M., Arévalo-Lomas L., Bolonio D., Barrio-Parra F., Izquierdo-Díaz M., Biosca \\ B., Sánchez-Palencia Y., Fernandez-GutierrezdelAlamo L \\ isabel.amez@upm.es, b.castells@upm.es,maria.scanales@upm.es, lucia.arevalo@upm.es, david.bolonio@upm.es, \\ fernando.barrio@upm.es, miguel.izquierdo@upm.es, barbara.biosca@upm.es, yolanda.sanchezpalencia@upm.es, \\ luis.fdezgda@gmail.com \\ Departamento de Energía y Combustibles \\ E.T.S.I. Minas y Energía (Universidad Politécnica de Madrid) \\ Madrid (España).
}

\begin{abstract}
Resumen- La crisis sanitaria producida por el virus del SARS-COV-2 produjo un cambio drástico en la educación en marzo de 2020, cuando la enseñanza presencial se vio forzada a adaptarse a la telenseñanza. Esto produjo cambios en las metodologías docentes, los materiales didácticos y los métodos de evaluación. El objetivo de este estudio es determinar cuáles de estos cambios, implementados en la Universidad presencial, han sido positivos y deberían mantenerse en el tiempo. Para ello se ha realizado una encuesta a los alumnos de los distintos cursos de los grados impartidos en la E.T.S.I. Minas y Energía (E.T.S.I.M.E) de la Universidad Politécnica de Madrid que permite conocer cómo ha influido la pandemia en el uso de los nuevos materiales didácticos, qué tipo de modalidades de enseñanza prefieren los alumnos y qué recursos utilizan más durante el estudio. A pesar de que los apuntes y diapositivas de clase siguen siendo el recurso predilecto de los alumnos, se ha visto un gran interés por las grabaciones de clases. Otros materiales más tradicionales, como la bibliografía o las tutorías, son menos utilizados por los estudiantes. En cuanto a las modalidades de enseñanza, la opinión está polarizada. Por tanto, es necesario realizar un esfuerzo para combinar eficientemente las metodologías y recursos didácticos de telenseñanza a la docencia presencial, generando recursos asíncronos sin perjuicio de las actividades presenciales tradicionales.
\end{abstract}

Palabras clave: Gestión de materiales, educación superior, COVID19, aprendizaje a distancia, encuesta al alumnado

Abstract- The health crisis caused by the SARS-COV-2 virus produced a drastic change in teaching in March 2020, when face-to-face teaching was forced to become online teaching. It led to changes in teaching methodologies, teaching materials and evaluation methods. The objective of this study is to determine which of these changes in the on-site university have been positive and should be sustained over time. To this end, a survey has been carried out among the students of the different courses of the degrees taught at the E.T.S.I. Minas y Energía (E.T.S.I.M.E) de la Universidad Politécnica de Madrid that allows us to know how the pandemic has influenced the use of didactic materials, what type of teaching modalities students prefer, and what resources they use the most during the study. Although class notes and slides remain the preferred resources among students, they showed great interest in class recordings. Other traditional materials, such as bibliography or tutoring, are less used by students. As for teaching methodologies, opinion is polarized. Therefore, it is necessary to make an effort to efficiently combine tele-teaching methodologies and didactic resources to face-to-face teaching, generating asynchronous resources without detriment to traditional face-to-face activities.

Keywords: Materials management, higher education, COVID-19, distance learning, student survey

\section{INTRODUCCIÓN}

La innovación educativa, como cualquier otro tipo de innovación, tiene como objetivo general cambiar y mejorar (Sein-Echaluce, Fidalgo-Blanco \& Aviles, 2017), es decir, fomenta cambios metodológicos y tecnológicos para desarrollar nuevos procesos de aprendizaje y/o nuevas formas de evaluación que sean mejores que los anteriores. Para realizar y validar la innovación es imprescindible realizar una planificación, establecer una previsión de los resultados y contar con indicadores con los que determinar el efecto del cambio producido (Fidalgo-Blanco, Sein-Echaluce \& GarciaPeñalvo, 2018).

La situación ideal de cambio sucede a través de la innovación educativa, con el objetivo de mejor la docencia, aunque desgraciadamente esto no siempre es así. En ese sentido, la situación de la educación general cambió de forma drástica en la primavera de 2020, cuando 188 países del mundo, entre ellos España, cerraron todas las aulas para evitar la propagación del COVID-19. Esto afectó al 91,3\% de la población estudiantil mundial. En una situación en la que los estudiantes no pueden asistir a clase presencialmente, la única alternativa es cambiar de la educación tradicional a la educación online (Basilaia \& Kvavadze, 2020).

Sin embargo, debido el COVID-19, la imposición de tener que pasar rápidamente de la impartición de docencia presencial a online no permitía una planificación por lo que, en la mayoría de los casos, solo supuso un cambio en la ubicación impartiendo la docencia a distancia y un incremento en el uso de las tecnologías de información y comunicación (TIC's), cuya implantación ya se venía llevando a cabo con anterioridad. Por 
lo tanto, desde un punto de vista pedagógico, la formación durante la pandemia no ha sido on-line, puesto que la inmediatez de respuesta no permitió la utilización de la metodología propia de la formación online ni la realización de una planificación previa al comienzo del curso, que son los dos ingredientes imprescindibles para que se pueda considerar formación on-line (Fidalgo-Blanco, 2020).

En cuanto a la digitalización, la pandemia ha favorecido el avance en las competencias digitales de la población, que se ha visto incrementado de forma exponencial. Sin embargo, concretamente en el ámbito universitario, que la aplicación de las tecnologías digitales haya permitido cumplir con las necesidades educativas, no significa que las instituciones, el profesorado y el alumnado estuvieran completamente preparados para realizar esta transformación digital impuesta por la situación, poniéndose de manifiesto las carencias existentes en este campo (García-Peñalvo, 2021).

Durante esta situación de emergencia vivida por el COVID19, era necesario dar respuesta instantánea y no había tiempo para comprobar la calidad y la eficacia del método de enseñanza adoptado, ya que el objetivo principal era salvar el proceso educativo y continuarlo en cualquier formato posible (Basilaia \& Kvavadze, 2020). Sin embargo, la nueva estrategia de enseñanza impuesta por la situación vivida no debería haber tenido como consecuencia peores resultados en el alumnado (George, 2020), por ello, es interesante averiguar qué metodologías aplicadas han dado como resultado el mismo (o mejor) desempeño del estudiante. En este sentido, es a posteriori, cuando se puede hacer un balance de lo realizado, extrayendo conclusiones sobre lo que puede y debe seguir utilizándose $\mathrm{y}$, por el contrario, lo que debería perfeccionarse para conseguir mejorar su eficacia.

\section{CONTEXTO}

La crisis sanitaria producida por el virus del SARS-COV-2 (COVID-19) obligó a cambiar drásticamente la modalidad de enseñanza de las universidades presenciales españolas. La mayor parte de ellas pasaron de una docencia eminentemente presencial a una docencia completamente a distancia y, en una segunda etapa, a un sistema "hibrido" semipresencial. Esta situación provocó cambios en la forma en la que los docentes impartían sus lecciones, generaban materiales docentes, evaluaban contenidos, etc. La consecuencia inmediata fue un cambio en la forma en que se desarrollaba el proceso enseñanza-aprendizaje. Algunos de los cambios introducidos han sido muy positivos y se prolongarán en los próximos años, sin embargo, otros han podido dificultar el aprendizaje. Algunos ejemplos de estas mejoras cuya implementación podría mantenerse a lo largo del tiempo son los sistemas de evaluación automática o el uso de plataformas digitales para agilizar entregas y correcciones. Para poder diferenciar los cambios metodológicos positivos, para seguir utilizándolos, de los negativos, para mejorarlos, es necesario conocer las impresiones y opiniones del alumnado. Analizar el público objetivo, en este caso el alumnado, permite elegir las opciones metodológicas que mejoren el proceso de enseñanzaaprendizaje en estudiantes con una predisposición cada vez mayor al consumo (a veces asíncrono) de contenidos audiovisuales y digitales en combinación con métodos de enseñanza presencial (lección magistral, clase de prácticas, problemas, etc.). Como avalan otras investigaciones, el alumnado es reticente a la docencia únicamente online, mayoritariamente prefiere la docencia presencial junto con otras herramientas online de apoyo (Aguado, 2020).

Por esta razón, se ha planteado una encuesta de opinión, mediante la cual se espera conocer los cambios que se han producido por la pandemia en el uso que dan los estudiantes a los materiales didácticos. Además, dada la incorporación de nuevas metodologías y recursos, se pretende determinar la acogida y percepción de utilidad por parte del alumnado. Estos materiales deben ser analizados también desde el punto de vista del aprendizaje. Los docentes disponen de tiempo limitado, por lo que es de vital importancia que dirijan sus esfuerzos a metodologías y materiales didácticos que van a ser realmente utilizados y aprovechados. Al mismo tiempo, se pretende discernir entre las medidas adoptadas por la situación sanitaria que deben mantenerse y aquellas que no producen mejoras en el aprendizaje.

La encuesta de opinión fue realizada en la E.T.S.I.M.E al grupo objetivo descrito en la tabla 1. Participan cuatro grados, Grado en I. de la Energía, I. de Minas, I. Geológica e I. de Recursos Energéticos, Combustibles y Explosivos.

Tabla 1 Tamaño de la muestra de la encuesta planteada

\begin{tabular}{cccccc}
\hline Curso & GIE & GITM & GIRECE & GIG & TOTAL \\
\hline 1ㅇ & 154 & 119 & - & - & 273 \\
2ㅇ & 211 & 107 & - & - & 318 \\
3ㅇ & 156 & 29 & 64 & 7 & 256 \\
4은 & 294 & 84 & 129 & 11 & 518 \\
\hline TOTAL & 815 & 339 & 193 & 18 & $\mathbf{1 3 6 5}$ \\
\hline
\end{tabular}

\section{DESCRIPCIÓN}

En la E.T.S.I.M.E, durante los años previos al 2020, aunque se habían ido incorporando métodos y tecnologías innovadores en muchas de sus asignaturas, predominaba un estilo de enseñanza tradicional. Por ejemplo, la Plataforma Moodle era ya esencial para la gestión de las asignaturas y para asegurar una comunicación dinámica entre el profesorado y el alumnado. Sin embargo, a pesar de ser esta la plataforma oficial de gestión, muchos profesores desconocen o tienen escaso conocimiento de la versatilidad de esta plataforma, y siguen optando por metodologías tradicionales en las clases magistrales, dejando de lado la operabilidad de la Moodle. Esta circunstancia está en consonancia con investigaciones anteriores, en las que se determinó que una baja capacitación tecnológica, unida al desconocimiento de parte del profesorado influyen en la baja frecuencia de utilización de algunas de las actividades interactivas disponibles por ejemplo en Moodle: como el chat, la wiki y el cuestionario, entre otros (Hernández, 2015). También es interesante destacar la formación dirigida hacia el profesorado en esta materia influye en un incremento exponencial del uso de estas herramientas (Andone, Ternauciuc y Vasiu, 2017). La crisis sanitaria que produjo la cancelación de las clases presenciales en marzo de 2020 desencadenó de forma abrupta que la enseñanza se desplazara del aula a las diferentes plataformas online. Esto implicó la necesidad de realizar una rápida adaptación de los materiales y herramientas disponibles que ayudara a los alumnos a continuar con el aprendizaje en una modalidad a distancia, obligando a los profesores a generar nuevos materiales en formato digital y a utilizar más estas herramientas que, algunos de ellos, habían utilizado poco hasta entonces o, incluso, que no habían utilizado nunca (como Teams, Zoom, BbCollaborate, etc.). 
A raíz de esta situación, los estudiantes tuvieron contacto por primera vez con algunos recursos digitales, como las clases online grabadas o asíncronas. Además, el cambio de docencia supuso también un cambio en la metodología de estudio del alumnado. Para conocer la opinión del alumnado, una vez que se ha normalizado la situación vivida, se optó por diseñar una encuesta de opinión a través de la cual se analizará la gestión de los materiales didácticos por parte del alumnado, antes y después de la pandemia, lo que dará como resultado una guía que podrá tenerse en cuenta en la preparación de los materiales didácticos y las metodologías a utilizar para los cursos venideros. La encuesta realizada se compone de tres bloques, que se describen a continuación:

Bloque 1 - Perfil del estudiante: Las preguntas de las que se compone este bloque (1-5) definirán el perfil del alumnado.

Bloque 2 - Gestión del material didáctico: Con las preguntas de este bloque (6-13) se pretende analizar de forma exhaustiva las diferencias percibidas por los alumnos antes y después de la pandemia del año 2020 en cuanto a la gestión de los materiales didácticos disponibles.

Bloque 3 - Análisis de materiales específicos y modalidades de docencia: En el último bloque, se busca conocer las preferencias generales del alumnado en referencia a la modalidad de la docencia, especialmente tras haber experimentado el cambio en la docencia del pasado año.

La encuesta fue respondida por alumnos de diversos cursos y titulaciones impartidas en la E.T.S.I. Minas y Energía (tabla 1) y pretende entender los cambios producidos por la pandemia en los materiales de estudio, además de conocer las opiniones de los alumnos referentes a las distintas modalidades de docencia, con el objeto de mejorar el aprendizaje.

\section{Resultados}

Los estudiantes que respondieron a la encuesta fueron 87 estando representados el alumnado de todos los grados impartidos en la E.T.S.I. Minas y Energía. La encuesta se divide en tres bloques cuyos resultados se muestran en las tres subsecciones siguientes.

\section{A. Bloque 1: Perfil de los alumnos}

Según las respuestas analizadas, el 66,7\% de los encuestados son hombres. Este resultado era de esperar al tratarse de grados técnicos. Los estudiantes comprenden edades entre 19 y 28 años, encontrándose el porcentaje mayoritario de los encuestados entre los alumnos nacidos en 2001 (20 años), con un $33 \%$ y que corresponden a alumnos de primer y segundo curso. El 64\% de los encuestados tenían una nota de acceso a la Universidad superior a 9. Por último, el 59,5\% de estudiantes tienen asignaturas pendientes de cursos anteriores, a pesar de que un tercio de los encuestados se encuentran entre el primer y el segundo curso. Además, el 65,9\% tiene pendiente al menos una asignatura de primero. Los resultados reflejan un mayor interés en responder por parte de los alumnos de segundo, ya que fueron los alumnos más afectados por el cambio de docencia consecuencia de la pandemia.

\section{B. Bloque 2: Gestión del material didáctico}

Los resultados de este bloque se exponen en función de las preguntas, que consideran dos escenarios: antes y después de la pandemia.
Preguntas 6 y 7: ¿Con cuanta frecuencia "consultabas y consultas ahora" el material didáctico proporcionado por el profesor en la plataforma Moodle?

Las respuestas reflejan un claro aumento en la frecuencia con la que los alumnos consultan el material didáctico en la plataforma Moodle. Mientras que antes de la pandemia el 58\% de los encuestados consultaba el material didáctico todas las semanas, este dato alcanza el $81 \%$ actualmente. Por el contrario, el porcentaje de alumnos que consultaba el material únicamente cuando tenía una duda ha bajado del $25 \%$ al $9,5 \%$ y los que únicamente lo consultaban antes del examen, se reduce desde el $12,5 \%$ al $6 \%$. El porcentaje de alumnos que no consultaban el material de forma frecuente (casi nunca o nunca), se mantiene prácticamente constante. Dichos alumnos que no consultan el material didáctico o no lo consultan casi nunca, indican que antes preferían consultar material didáctico de fuentes externas distintas al profesor, y tras la pandemia, el recurso que consideran más interesante es la bibliografía. Todos ellos además acuden a los compañeros para resolver dudas.

Preguntas 8 y 9: ¿Qué tipo de material didáctico “consultabas y consultas ahora" con más frecuencia?

Las preguntas 8 y 9 comparan el tipo de material didáctico consultado por los alumnos antes y después de la pandemia. Como puede verse en la figura 1 a), los porcentajes prácticamente no varían, por lo que el cambio de docencia no ha afectado prácticamente al tipo de material consultado por los alumnos. No obstante, sí se aprecia un descenso en la consulta de apuntes y diapositivas de clase, y un aumento en el material complementario (tanto el aportado por el profesor como el de otras fuentes). La bibliografía se mantiene en ambos casos como el material menos consultado, registrando únicamente un $3-4 \%$. Analizando las respuestas, se ha visto que este porcentaje coincide con los alumnos que no consultan el material aportando por el profesor nunca o casi nunca.

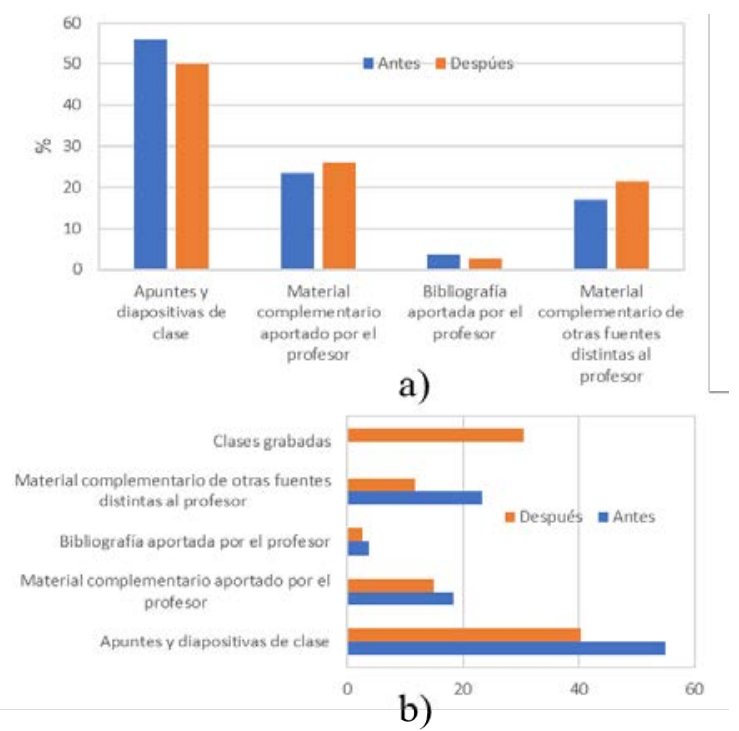

Figura 1 a) Respuestas a preguntas 8 y 9 (material consultado); b) Respuestas a preguntas 10 y 11 (material más interesante)

Preguntas 10/11: ¿Qué tipo de material didáctico "considerabas y consideras ahora" más interesante para consultar? 
Lógicamente, los alumnos consultan los materiales que consideran más interesantes, por ello cuando se pregunta qué material se considera más interesante para consultar (preguntas 10 y 11), las respuestas concuerdan con las ofrecidas en las preguntas 7 y 8 . Aunque se pueden observar ligeras diferencias, la tendencia coincide completamente, especialmente en la pregunta referida a la situación prepandemia. Sin embargo, en la pregunta 10 se ha querido añadir la opción de "clases grabadas" para comprobar el interés real que existe en este tipo de contenido. Aunque este tema se aborda en una pregunta concreta más adelante, la figura 1 b) ya denota el interés de los estudiantes en las clases grabadas. Tanto es así, que el porcentaje se acerca sensiblemente al de los apuntes y diapositivas de clase, que se mantienen como materiales predominantes.

Preguntas 12 y 13: En caso de detectar una duda durante el estudio, ¿Qué medida tomabas de entre las siguientes antes de la pandemia? / ¿Qué medida tomas ahora de entre las siguientes?

Las preguntas 12 y 13 pretenden determinar a qué fuentes acuden los estudiantes cuando encuentran una duda o dificultad durante el estudio de los contenidos y si, el hecho de haber recibido las clases a distancia con un contacto menos personal con el profesorado y con sus compañeros, ha podido influir en el descenso de las consultas entre compañeros y con el profesor en las tutorías. En la figura puede verse como los cambios más significativos se centran en la consulta a los compañeros y la consulta de vídeos y tutoriales facilitados por el profesor (siendo 1 la situación anterior y 2 la situación actual). Antes de la pandemia, los alumnos consultaban con mayor frecuencia a sus compañeros, habiéndose disminuido este porcentaje un $16,3 \%$. Esta situación es fácil de explicar ya que la situación sanitaria ha obstaculizado las relaciones sociales, sin embargo, esta circunstancia no ha reducido las tutorías con el profesorado. Por otro lado, se observa un incremento del $28 \%$ en la consulta de vídeos y videotutoriales aportados por el profesor.

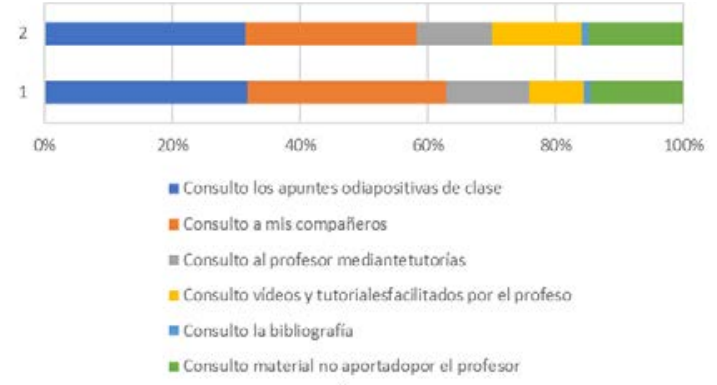

a)

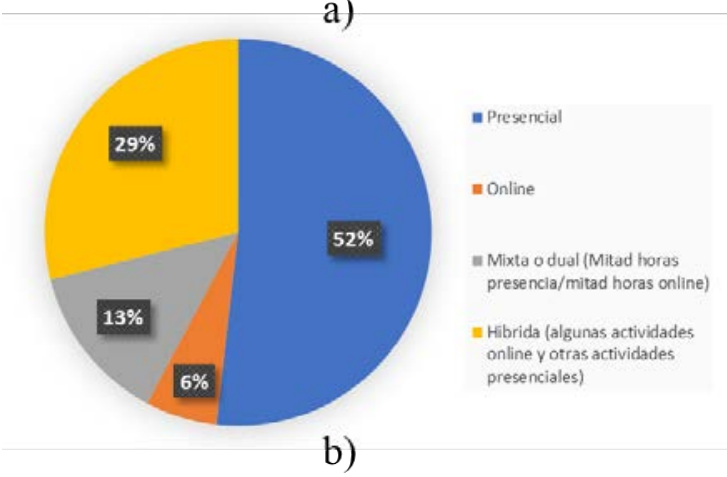

b)

Figura 2 a) Respuestas a preguntas 12 y 13 (medidas frente a dudas); b) Respuesta a pregunta 14 (modelo de docencia)

\section{Bloque 3: Análisis de materiales específicos y modalidades de docencia}

Pregunta 14: Si tuvieras que elegir un único modelo de docencia, ¿Cuál sería?

La figura 3 b) recoge las preferencias de los estudiantes encuestados en cuanto a la modalidad de docencia, mostrando una gran preferencia por la modalidad presencial. Sin embargo, este dato (52\%) apenas supera la mitad de las respuestas. Destaca el alto porcentaje de estudiantes que prefieren una enseñanza híbrida, que en el presente estudio se ha definido como una docencia combinada, en la que algunas actividades se hacen de forma presencial y otra vía online. Cuando se compara con la modalidad mixta o dual (mitad de la docencia presencial y mitad online) este porcentaje se divide a la mitad. De estos datos se puede extrapolar la polarización de opiniones entre la modalidad presencial y la combinada (tanto híbrida como mixta).

Pregunta 15: ¿Dirías que la situación sanitaria provocada por la Covid ha mejorado los contenidos y materiales en la plataforma Moodle?

Pregunta 16: ¿Consultas ahora con mayor frecuencia los contenidos colgados en Moodle?

Cuando se pregunta los estudiantes si el cambio de docencia provocado por la situación sanitaria ha mejorado los contenidos y materiales en la plataforma Moodle, el 62,5\% opina que sí (pregunta 15). Este hecho es significativo, y es una consecuencia positiva que se ha observado tras la pandemia, ya que los profesores (al carecer de docencia presencial) se han visto obligados a aumentar y mejorar los contenidos adicionales. Además, aunque este dato ya se ha visto reflejado en preguntas anteriores, la pregunta 16 confirma que el 80,5\% de los alumnos consultan ahora con mayor frecuencia los contenidos disponibles en Moodle.

Pregunta 17: ¿Te parece interesante que se graben las clases?

Pregunta18: ¿Por qué?

Las preguntas 17 y 18 buscan analizar el interés de los alumnos en las clases grabadas, y conocer las causas por las cuales el alumnado lo considera un recurso valioso. Para ello se añadió la pregunta 19, una pregunta abierta en la que se obtuvieron 75 respuestas y que se analizan a continuación. Casi el total de los encuestados (un 96,4\%) considera interesante que se graben las clases, pero ¿cuáles son las razones?

Muchas de las respuestas se enfocan en el caso concreto de la docencia online, ya que les resulta difícil seguir las clases en modalidad online, y tenerlas grabadas facilita el aprendizaje. Según explican los alumnos, y como ya se ha comprobado en estudios recientes, la concentración en las clases online es difícil de mantener. Este resultado está en consonancia con un estudio reciente en el que, entre las dificultades experimentadas por el alumnado para continuar los estudios de forma telemática impuesto por el COVID-19, entre las tres más importantes, se encuentran que "en casa no me concentro" y que "me resulta difícil seguir las clases o tutorías online" (Kuric, CalderónGómez y Sanmartín Ortí , 2021) pero la pérdida de concentración no solo sucede en la docencia online, en estudios anteriores a la pandemia, también se constató que el alumnado perdía la concentración en las clases presenciales por diferentes factores: por los compañeros, por condiciones del ambiente y, principalmente, por el uso del móvil durante las clases 
(Mendoza, 2018). Ya sea durante las clases online o presenciales la pérdida de concentración por parte del alumnado disminuye su capacidad de aprendizaje y, por tanto, supone una disminución en su adquisición de las competencias. Por ello, el alumnado considera que la posibilidad de disponer de las clases y poder verlas de nuevo, les permite repetir aquellas explicaciones más complejas permitiéndoles comprender los conceptos más complicados.

Además de lo comentado anteriormente, el 54,9\% de las respuestas se enfocan en la utilizada de las clases grabadas como recurso educativo adicional, que sería válido incluso para las clases presenciales. El método de enseñanza con videotutoriales o videos explicativos, de problemas, etc., es una técnica extensamente implantada (Castells et al., 2019). Sin embargo, el forzoso cambio de docencia experimentado en el 2020 ha despertado el interés de este tipo de recursos que los alumnos anteriormente no consultaban con tanta frecuencia. Dado que la mayoría de los alumnos prefieren docencia presencial, sería interesante potenciar este tipo de recursos, especialmente para aquellos conceptos en los que se detecten errores más comunes.

Además, un $21,1 \%$ de los estudiantes confirman la importancia de acceder a este tipo de recursos cuando existen incompatibilidades de horarios.

Únicamente un 4,2\% de las respuestas destacan aspectos negativos en referencia al uso de las clases grabadas. Según estos alumnos, ser conocedores de que las clases van a ser grabadas, puede ocasionar perdida de atención en las clases. Es interesante mencionar algunas respuestas, como la posibilidad de que cada alumno estudie a su propio ritmo.

\section{Pregunta 19: ¿Qué metodologías de estudio prefieres?}

La pregunta 19 plantea la cuestión de la enseñanza online o presencial, desde el punto de vista de la metodología de estudio preferida por los alumnos. Como se ve en la figura 3, sigue existiendo un alto porcentaje de alumnos que prefieren trabajar de forma tradicional, mediante clases presenciales. Es de entender, que los apuntes propios se pueden utilizar en ambas modalidades. De nuevo, destaca el alto porcentaje que prefiere estudiar con clases online grabadas $(23,5 \%)$. A la vista de los resultados, parece claro que los estudiantes actuales ven de gran utilidad el poder acceder a explicaciones grabadas, lo que fomenta que todos los alumnos sean capaces de seguir el curso, dependiendo de su ritmo de aprendizaje. Este modelo no es del todo aplicable a los sistemas de enseñanza presenciales, pero sí deja abierta la puerta a materiales didácticos de apoyo, en formato vídeo, en los que se presenten explicaciones de conceptos específicos. También se podrían utilizar metodologías activas como el Flip teaching (o aula invertida) que se basa en invertir lo que se hace en clase y lo que se hace fuera de clase con respecto a la enseñanza tradicional, es decir, en el Aula invertida los estudiantes reciben la teoría en casa (que puede ser en forma de videos) y luego trabajan en clase con las actividades propuestas (Nouri, 2016). Se ha demostrado que esta metodología aplicada en la docencia universitaria conlleva muchas ventajas, por ejemplo, permite que los estudiantes aprendan a su propio ritmo, alienta a los estudiantes a participar activamente, libera tiempo real de clase para más actividades de aprendizaje eficaces, creativas y activas, los profesores reciben más oportunidades para interactuar y evaluar el aprendizaje de los estudiantes, y los estudiantes asumen el control y la responsabilidad de su aprendizaje (Gilboy,
Heinerichs y Pazzaglia, 2015; Betihavas et al., 2015). Además, en la figura 3 se refleja que no existe unanimidad por parte de los alumnos en cuanto a las metodologías de estudios preferidas, lo que indica una necesidad de docencia adaptada al estudiante. Esta situación podría ser subsanada con materiales didácticos adecuados que permitan el seguimiento de las clases de aquellos alumnos con diferentes ritmos.

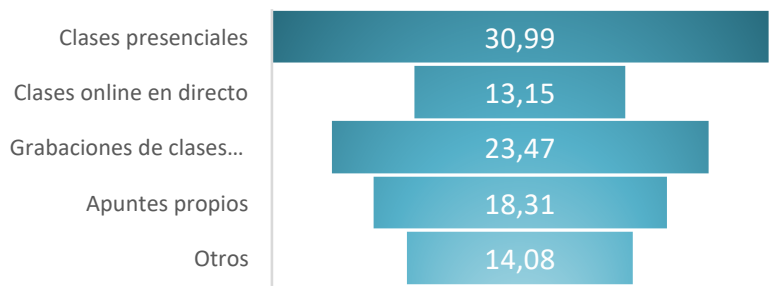

Figura 3: Respuesta a la pregunta 19 (material de estudio)

Pregunta 20: Consideras el material proporcionado por el profesor en la situación actual es:

Finalmente, se plantea la pregunta 20 en la que se pidió a los estudiantes que valoraran la cantidad de contenidos aportados por el profesor en Moodle. El 67,5\% de los estudiantes cree que el contenido es suficiente, es decir, consideran que tienen a su disposición todos los materiales necesarios para adquirir las competencias de sus asignaturas. Es preocupante que un 26,5\% de los alumnos consideren que no disponen de los materiales suficientes. El $6 \%$ restante, considera que el material es excesivo. No obstante, a pesar de que los alumnos, en su mayoría; consideran suficiente el material disponible, muchos consideran que la organización de los mismos no es correcta. Muchas respuestas inciden en el material mal organizado que les impide determinar qué materiales son más importantes o básicos, y cuáles son más secundarios y adicionales.

\section{CONCLUSIONES}

La crisis sanitaria no sólo ha producido cambios en la docencia, sino que también ha supuesto un cambio en la gestión de los alumnos del material didáctico. A pesar de que los apuntes y diapositivas de clase siguen siendo el material más utilizado, el cambio de docencia ha producido un incremento notable en la consulta de materiales adicionales tanto aportados por el profesor como de fuentes externas, produciendo un aumento en el aprendizaje autónomo del alumno. La bibliografía es el material que menor interés despierta en los alumnos, lo que concuerda con su preferencia por materiales interactivos o visuales.

En este sentido, cabe destacar el interés por las clases grabadas como material de estudio, que solventa problemas como distintos ritmos de aprendizaje entre alumnos o posibles incompatibilidades de horarios. Además, se crean nuevos materiales didácticos en formato vídeo enfocados a la resolución de problemas y dudas que sirvan no sólo durante el aprendizaje guiado sino también durante el aprendizaje autónomo del alumno.

Durante este periodo también se ha producido un descenso en la resolución de dudas mediante consultas a compañeros al reducirse la interacción a contactos a través de Internet principalmente. No obstante, cabe destacar que esta opción sigue manteniéndose como la segunda preferida (por detrás de 
la consulta de apuntes o diapositivas de clase) muy por encima de las tutorías, que ha seguido con la misma baja incidencia que antes de la pandemia. Con el cambio a la enseñanza a distancia los alumnos disponían de medios más inmediatos y accesibles para asistir a tutorías (chats y vídeo llamadas) y, sin embargo, no han utilizado este recurso.

En lo referente a la modalidad de enseñanza, se ha visto una preferencia entre los alumnos por la modalidad presencial (52\%) y, casi a la par, la combinación de enseñanza presencial y telenseñanza (42\%). Dentro de este último grupo, destaca la preferencia por la combinación de actividades presenciales y a distancia. De ello se desprende que los alumnos consideran que las actividades presenciales fomentan el aprendizaje, pero todo apunta a que en un futuro será necesario incorporar más actividades a distancia. En este sentido, es muy bajo el porcentaje de alumnado que prefiere solo docencia a distancia $(6 \%)$, entendible con el hecho de que la universidad en la que estudian es presencial, por lo que, de preferir solo docencia online podrían optar por otras universidades que la ofrecen.

Por último, la crisis sanitaria ha obligado a aumentar y mejorar los recursos que ponen los docentes a disposición de los alumnos. Las encuestas revelan que la mayor parte de los alumnos consideran que la cantidad de recursos es en general adecuada, no obstante, reclaman una mayor organización de estos, y pautas que les ayuden a entender la importancia y utilidad de cada uno de los materiales disponibles. Para ello, cuando se dispone de mucho material, se podrían crear repositorios aplicar y utilizar mecanismos de búsqueda, que ayudaría a conocer mejor el material y localizarlo más fácilmente (Fidalgo et al. 2018).

Por lo tanto, este estudio preliminar, puede servir como orientación para los docentes, en primera instancia, y para los órganos de gobierno de las universidades presenciales, para mantener las estrategias y acciones que les han resultado más útiles a los estudiantes en su aprendizaje durante esta situación excepcional. A modo de ejemplo de lecciones aprendidas, se podría llevar a cabo una docencia presencial combinada de forma puntual con actividades online síncronas que podrían ser grabadas para su posterior consulta por parte del alumnado. Otra acción a realizar sería la de mejorar la organización y clasificación del material docente digital que ha aumentado considerablemente. Por último, la falta o escasa interacción interpersonal durante los dos cursos académicos pasados entre el alumnado podría repercutir en una disminución en la resolución de dudas entre compañeros por lo que se sugiere hacer especial hincapié en la recuperación de estos vínculos en la era postcovid.

\section{REFERENCIAS}

Aguado Franco, J. C. (2020). Los MOOC: ¿sustituto o complemento de la formación tradicional? Revista Tecnología, Ciencia Y Educación, (16), 41-62. doi:10.51302/tce.2020.439

Andone, D., Ternauciuc, A., \& Vasiu, R. (2017). Using Open Education Tools for a Higher Education Virtual Campus. In 17th IEEE International Conference on Advanced Learning Technologies (ICALT), 26-30.

Basilaia, G., \& Kvavadze, D. (2020). Transition to Online Education in Schools during a SARS-CoV-2 Coronavirus (COVID-19) Pandemic in Georgia. Pedagogical Research, 5(4), 1-9. doi: $10.29333 / \mathrm{pr} / 7937$
Betihavas, V., Bridgman, H., Kornhaber, R., \& Cross, M. (2015). The evidence for 'flipping out': A systematic review of the flipped classroom in nursing education. Nurse Education Today, 6, 1521.

Castells, B., Biosca, B., Amez, I., Izquierdo-Díaz, M., Barrio-Parra, F., Sánchez-Palencia, Y., Bolonio, D., Sánchez-Canales, M., Valiño, V., Montalvo, C. \& Fernández-GutiérrezdelAlamo, L. (2019). Vídeo-tutoriales y su influencia en el aprendizaje (No. COMPON-2019-CINAIC-0062).

Fidalgo-Blanco, Á., Sein-Echaluce, M. L., \& García-Peñalvo, F. J. (2018). Method for Applying Innovation in educativo Proceedings TEEM'18. Sixth International Conference on Technological Ecosystems for Enhancing Multiculturality (pp. 806-813). New York, NY, USA: ACM. doi: $10.1145 / 3284179.3284313$

Fidalgo-Blanco, Á. (2020) “¿Qué se podría haber hecho, y no se ha hecho, con la innovación educativa durante el confinamiento por el COVID-19?," Blog Innovación Educativa, 2020. [Online]. Available:

https://innovacioneducativa.wordpress.com/2020/04/20/que-sepodria-haber-hechoy-no-se-ha-hecho-con-la-innovacioneducativa-durante-el-confinamiento-por-el-covid19/. [Accessed: 10-May-2021].

Fidalgo-Blanco, Á., Sánchez-Canales, M., Sein-Echaluce, M. L., \& García-Peñalvo, F. J. (2018). Ontological Search for Academic Resources. Proceedings TEEM'18. Sixth International Conference on Technological Ecosystems for Enhancing Multiculturality, 788-793. New York, NY, USA: ACM. doi: $10.1145 / 3284179.3284315$

García-Peñalvo, F. J. (2021). Digital Transformation in the Universities: Implications of the COVID-19 Pandemic / Transformación digital en las universidades: Implicaciones de la pandemia de la COVID-19. Education in the Knowledge Society (EKS). 22. e25465. doi:10.14201/eks.25465.

George, M. L. (2020). Effective teaching and examination strategies for undergraduate learning during COVID-19 school restrictions. Journal of Educational Technology Systems, 49(1), 23-48.

Gilboy, M. B., Heinerichs, S., \& Pazzaglia, G. (2015). Enhancing student engagement using the flipped classroom. Journal of nutrition education and behavior, 47(1), 109-114.

Hernández, G. (2015). Análisis del uso y manejo de la plataforma Moodle en docentes de matemáticas, para el desarrollo de competencias integrales en estudiantes de primaria. Revista Q, 10 (19). doi:10.18566/rev istaq.v10n19.a01

Kuric Kardelis, Stribor; Calderón-Gómez, Daniel y Sanmartín Ortí, Anna (2021). Educación y brecha digital en tiempos del COVID-19. Perfiles y problemáticas experimentadas por el alumnado juvenil para afrontar sus estudios durante el confinamiento. Revista de Sociología de la Educación-RASE, 14 (1), 63-84. doi:10.7203/RASE.14.1.18265

Mendoza Osuna, Henry Mauricio. (2018). Gestión de competencias en carreras del área empresarial, a través de la neuroeducación. Revista Investigación y Negocios, 11(17), 30-46.

Nouri, J. (2016). The flipped classroom: for active, effective and increased learning - especially for low achievers. Int J Educ Technol High Educ 13, 33. doi:10.1186/s41239-016-0032-z

Sein-Echaluce, M. L., Fidalgo-Blanco Á. \& Alves, G. (2017) "Technology Behaviors in Education Innovation," Computers in Human Behavior, 72. 596-598. doi: 10.1016/j.chb.2016.11.049 